\title{
Fluctuation-induced pair density wave in itinerant ferromagnets
}

\author{
G. J. Conduit, ${ }^{1, *}$ C. J. Pedder, ${ }^{2}$ and A. G. Green ${ }^{2, \dagger}$ \\ ${ }^{1}$ Cavendish Laboratory, University of Cambridge, CB3 OWA, United Kingdom \\ ${ }^{2}$ London Centre for Nanotechnology, University College London, 17-19 Gordon Street, London, WC1H 0AH, United Kingdom
}

(Received 1 December 2012; revised manuscript received 17 March 2013; published 27 March 2013)

\begin{abstract}
Magnetic fluctuations near to quantum criticality can have profound effects. They lead to characteristic scaling at high temperature which may ultimately give way to a reconstruction of the phase diagram and the formation of new phases at low temperatures. The ferromagnet $\mathrm{UGe}_{2}$ is unstable to $p$-wave superconducting order-an effect presaged by the superfluidity in ${ }^{3} \mathrm{He}$ - whereas in $\mathrm{CeFePO}$ fluctuations drive the formation of spiral magnetic order. Here we develop a general quantum order-by-disorder description of these systems that encompasses both of these instabilities within a unified framework. This allows us to demonstrate that in fact these instabilities intertwine to form a pair density wave.
\end{abstract}

DOI: 10.1103/PhysRevB.87.121112 PACS number(s): 74.40.Kb, 71.10.-w, 74.20.-z, 75.30.Kz

\section{INTRODUCTION}

The physics of metallic systems tuned near to continuous, zero-temperature phase transitions has provided a rich vein of experimental surprises and theoretical insights. ${ }^{1-3}$ Fluctuations in such systems drive new critical behavior that inextricably involves quantum dynamics, described by $\mathrm{Hertz}^{4}$ as quantum criticality. Over the past decade, it has become apparent that quantum fluctuations have an even more profound effect on the magnetic phase diagram. ${ }^{5-10} \mathrm{~A}$ breakdown of the standard Moriya-Hertz-Millis ${ }^{4,11,12}$ model of itinerant magnetic criticality occurs as the quantum critical point becomes occluded by new fluctuation-driven phases. Magnetic fluctuations transverse to the polarization drive many materials to display a first-order ferromagnetic transition, ${ }^{13}$ spatially modulated magnetic order-for example, $\mathrm{CeFePO},{ }^{14} \mathrm{NbFe}_{2},{ }^{15}$ $\mathrm{Yb}\left(\mathrm{Rh}_{0.73} \mathrm{Co}_{0.27}\right)_{2} \mathrm{Si}_{2},{ }^{16}$ and possibly $\mathrm{Sr}_{3} \mathrm{Ru}_{2} \mathrm{O}_{7},{ }^{17,18}$ or higher angular momentum particle-hole pairing. ${ }^{19,20}$ Moreover, in materials such as $\mathrm{UGe}_{2},{ }^{21-23} \mathrm{URhGe}^{24}$ and $\mathrm{UCoGe},{ }^{25,26}$ longitudinal magnetic fluctuations can drive $p$-wave Cooper pairing in a similar manner to ${ }^{3} \mathrm{He}$ superfluidity. ${ }^{27-29}$

In this Rapid Communication we study the minimal model of freely dispersing electrons interacting through a contact repulsion. We develop a general quantum order-by-disorder approach to calculate the effects of fluctuations on the free energy, leading to the phase diagram in Fig. 1. Near to the itinerant ferromagnetic quantum critical point the electron gas forms a pair density wave where the magnetic order forms a spiral modulation coexistent with a Larkin-OvchinnikovFulde-Ferrell-like spatial modulation of the $d$ vector of a $p$ wave superconductor. ${ }^{30-32}$

The essence of our order-by-disorder approach is to expand the free energy in fluctuations about some putative order. The order modifies the electron dispersion and so the energy of the quantum fluctuations. This offers an alternative, physical view of the nonanalytic corrections that overturn Moriya-HertzMillis theory leading to the opportunity to undergo a firstorder transition, ${ }^{5,6,13,33,34}$ spatially modulated magnetism, ${ }^{35,36}$ a nematic phase, ${ }^{20}$ and $p$-wave superconductivity. ${ }^{37-41} \mathrm{Near}$ to the quantum critical point the fluctuations self-consistently stabilize the ordered state. This mechanism is similar in spirit, but subtly different in details, to several others: the ColemanWeinberg mechanism of field theory, ${ }^{42}$ quantum order by

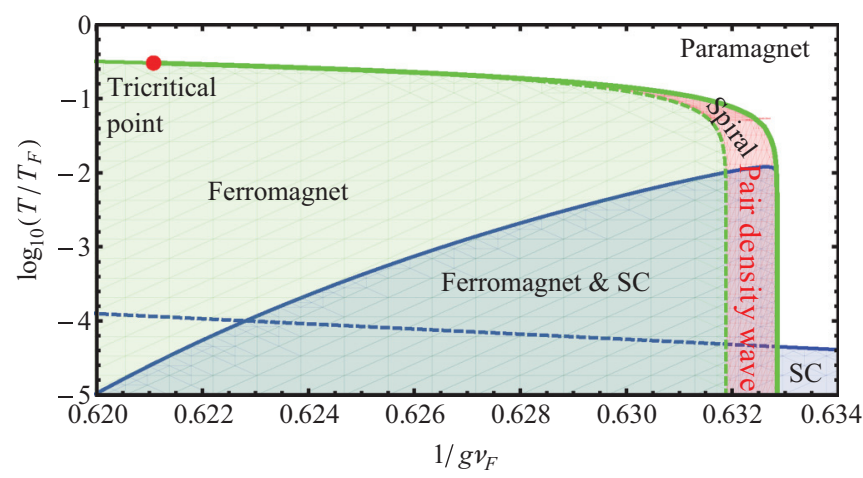

FIG. 1. (Color online) The phase diagram of Hamiltonian, Eq. (1), as a function of temperature and interaction strength. Our phase, the pair density wave, is highlighted by the red text. $g$ is the interaction strength, $\nu_{\mathrm{F}}$ is the density of states at the Fermi surface, $T$ temperature, and $T_{\mathrm{F}}$ the Fermi temperature. The magnetic background to this phase diagram is as determined in Ref. 35, allowing for a resummation of the leading divergent contributions to the free energy. At high temperatures, there is a continuous transition from ferromagnet to paramagnet. The temperature of this reduces with decreasing interaction strength until the red tricritical point. At this point, there is a Lifshitz transition from the ferromagnet into a magnetic spiral (green dotted line). Finally, the spiral magnetic order gives way to paramagnetism in a first-order transition. Superconductivity in the absence of magnetic order occurs below the blue dotted line. This transition temperature rises dramatically in the presence of magnetic order due to mode-mode coupling. Where superconductivity overlaps, the spiral magnetic order a Larkin-Ovchinnikov-Fulde-Ferrell-like pair density wave state is formed, highlighted by the red text.

disorder, ${ }^{43}$ and the Casimir effect. ${ }^{44}$ From the latter perspective, the Fermi surface may be viewed as providing boundary conditions for quantum fluctuations through Pauli exclusion. Modifying the Fermi surface by the introduction of order modifies the spectrum of the fluctuations. This perspective emphasizes the central role played by the underlying fermionic statistics (which is absent in the conventional application of these ideas). However, we have chosen to use the term quantum order by disorder to be closest in spirit to the conventional usage. 
In this Rapid Communication we first revisit the quantumby-disorder approach, demonstrating how transverse magnetic fluctuations can generate a spatially modulated magnetic phase and extend the analytical formalism to apply near zero temperature. Second, we introduce a superconducting instability driven by longitudinal spin fluctuations into the formalism and elucidate the phase diagram. Finally, we discuss the consequences of the intertwined spiral and superconducting instabilities for experimental systems.

\section{QUANTUM ORDER BY DISORDER IN THE ITINERANT FERROMAGNET}

We first review the order-by-disorder approach to address the magnetic phase diagram, ignoring any possible superconducting instabilities. Our starting point is a Hamiltonian of freely dispersing electrons with $\epsilon_{\mathbf{k}}=k^{2} / 2$ interacting through a contact repulsion $g$ reflecting the screened Coulomb interaction present in metals,

$$
\mathcal{H}=\underbrace{\sum_{\mathbf{k}, \sigma} \epsilon_{\mathbf{k}} \hat{n}_{\mathbf{k}}^{\sigma}}_{\mathcal{H}_{0}}+\underbrace{g \int d \mathbf{r} \hat{n}_{\mathbf{r}}^{\uparrow} \hat{n}_{\mathbf{r}}^{\downarrow}}_{\mathcal{H}_{\text {int }}} .
$$

Here $\hat{n}_{\mathbf{k}}^{\sigma}$ is the fermion occupancy in momentum space, $\hat{n}_{\mathbf{r}}^{\sigma}$ in real space, spin $\sigma \in\{\uparrow, \downarrow\}$, and we adopt atomic units with $\hbar=m=1$ throughout. This model shows a continuous ferromagnetic transition in mean field, the zero-temperature terminus of which Hertz termed the quantum critical point. ${ }^{4}$ Hertz realized that quantum fluctuations could modify the critical behavior at this quantum critical point, however, it has recently been shown that fluctuations can have an even more dramatic effect, reconstructing the phase diagram. ${ }^{5,7-10,33,35,36}$

The analysis of these effects proceeds as follows: First we consider a background spiral magnetization, $\mathbf{M}_{\mathbf{q}}=$ $M(\cos \mathbf{q} \cdot \mathbf{r}, \sin \mathbf{q} \cdot \mathbf{r}, 0)$, that modifies the mean-field electron dispersion $\epsilon_{\mathbf{k}}^{\sigma}\left(\mathbf{M}_{\mathbf{q}}\right)=\epsilon_{\mathbf{k}}+\sigma \sqrt{(\mathbf{k} \cdot \mathbf{q})^{2}+(g M)^{2}}$. The meanfield and fluctuation corrections to the free energy are then calculated in the presence of this background spiral. To leading order in the fluctuations, the free energy is given by $\mathcal{F}=\mathcal{F}_{\text {MF }}+\mathcal{F}_{\text {fluct }}$ with

$$
\mathcal{F}_{\mathrm{MF}}=-T \sum_{\sigma, \mathbf{k}} \ln \left[e^{\left(\epsilon_{\mathbf{k}}^{\sigma}\left(\mathbf{M}_{\mathbf{q}}\right)-\mu\right) / T}+1\right]+\frac{g}{2} M_{\mathbf{q}}^{2},
$$

where $\mu$ is the chemical potential, $T$ temperature, and

$$
\mathcal{F}_{\text {fluct }}=g^{2} \sum_{\substack{\mathbf{k}_{1}+\mathbf{k}_{2}=\mathbf{k}_{3}+\mathbf{k}_{4} \\ \mathbf{p}_{1}+\mathbf{p}_{2}=\mathbf{p}_{3}+\mathbf{p}_{4} \\ s}} \frac{\left\langle c_{\mathbf{k}_{1} \uparrow}^{\dagger} c_{\mathbf{k}_{2} \downarrow}^{\dagger} c_{\mathbf{k}_{3} \downarrow} c_{\mathbf{k}_{4} \uparrow} \mid s\right\rangle\left\langle s \mid c_{\mathbf{p}_{1} \uparrow}^{\dagger} c_{\mathbf{p}_{2} \downarrow}^{\dagger} c_{\mathbf{p}_{3} \downarrow} c_{\mathbf{p}_{4} \uparrow}\right\rangle}{\epsilon_{\mathbf{k}_{1}}^{\uparrow}+\epsilon_{\mathbf{k}_{2}}^{\downarrow}-\epsilon_{\mathbf{k}_{3}}^{\uparrow}-\epsilon_{\mathbf{k}_{4}}^{\downarrow}} .
$$

The expectation is taken in the thermal ensemble and the states $|s\rangle$ are virtual intermediate states generated by the action of the Hamiltonian. The fluctuation contribution to the free energy is then calculated following the prescription of Refs. 33 and 34, with the result ${ }^{45}$

$$
\mathcal{F}_{\text {fluct }}=-2 g^{2} \sum_{\substack{\mathbf{k}_{1}+\mathbf{k}_{2} \\=\mathbf{k}_{3}+\mathbf{k}_{4}}} \frac{n_{\mathbf{k}_{1}}^{\uparrow} n_{\mathbf{k}_{2}}^{\downarrow}\left[n_{\mathbf{k}_{3}}^{\uparrow}+n_{\mathbf{k}_{4}}^{\downarrow}\right]}{\epsilon_{\mathbf{k}_{1}}^{\uparrow}+\epsilon_{\mathbf{k}_{2}}^{\downarrow}-\epsilon_{\mathbf{k}_{3}}^{\uparrow}-\epsilon_{\mathbf{k}_{4}}^{\downarrow}} .
$$

Reference 35 evaluated the $\mathcal{F}_{\text {fluct }}$ numerically at each value of $\mathbf{M}_{\mathbf{q}}$. This revealed that the low-energy phase space of fluctuations is enhanced by uniform or spiral magnetic order. Good agreement with variational Monte Carlo calculations ${ }^{35}$ encourages us to develop a more amenable Ginzburg-Landau expansion near to the tricritical point. ${ }^{20}$ The free energy is a functional of the mean-field dispersion in the presence of the modulated order parameter, $\mathcal{F}\left(M_{\mathbf{q}}\right) \equiv \mathcal{F}\left[\epsilon_{\mathbf{k}}\left(\mathbf{M}_{\mathbf{q}}\right)\right]=$ $\left\langle\mathcal{F}\left[M^{2}+\theta^{2} q^{2}\right]\right\rangle-\left\langle\mathcal{F}\left[\theta^{2} q^{2}\right]\right\rangle$, where $\theta=\mathbf{k} \cdot \mathbf{q} /|\mathbf{k}||\mathbf{q}|$ and $\langle\cdots\rangle$ indicates an angular average. In a Taylor expansion of the free energy in $M^{2}$ and $q^{2}$, terms are then linked by geometric factors stemming from the angular average

$$
\mathcal{F}=\left(\alpha+\frac{2}{3} \beta q^{2}+\frac{2}{5} \gamma q^{4}\right) M^{2}+\left(\beta+\gamma q^{2}\right) M^{4}+\gamma M^{6}
$$

so the expansion contains only three coefficients $\alpha, \beta$, and $\gamma$ that are functions of the interaction strength $g$ and the temperature $T$. Reconstruction of the phase diagram is driven by a $\log T$ contribution to the $M^{4}$ coefficient $\beta$. The analytical form of these parameters is known near to the tricritical point. ${ }^{20}$

We now seek a superconducting instability that occurs at very low temperatures away from the tricritical point. In order to access this, we must extend the region of validity of our expansion to lower temperatures away from the vicinity of the tricritical point. The leading fluctuation contribution, $M^{4} \ln T$, is accompanied by higher-order terms proportional to $M^{6} / T^{2}$, $M^{8} / T^{4}$, etc. These occur with prefactors allowing them to be resummed exactly to give the following contribution to the free energy,

$$
\frac{\nu_{\mathrm{F}}\left(g \nu_{\mathrm{F}}\right)^{2}}{6} \frac{(g M)^{4}}{\mu^{2}} \ln \left[\frac{(g M)^{2}+\left(\pi T / 4 e^{\mathcal{C}}\right)^{2}}{4 \mu^{2}}\right]
$$

where $\nu_{\mathrm{F}}$ is the density of states at the Fermi surface and $\mathcal{C}$ is the Euler constant. This is consistent with that found in previous studies. ${ }^{5,8,35}$ In practice, subleading terms that are less divergent in $T$ have an important effect upon the position of the phase boundaries at low temperatures away from the tricritical point. We find that they enhance the region of the phase diagram occupied by the spiral phase, making it consistent with the broader region seen in variation Monte Carlo calculations. ${ }^{35}$ Unfortunately, resummation of these subleading divergences does not yield a simple analytical form and we have not included them here.

\section{SUPERCONDUCTIVITY IN QUANTUM ORDER BY DISORDER}

We now wish to incorporate the possibility of Cooper pairing. We focus upon A1 type pairing of spin-up electrons into a $p$-wave state, ${ }^{46}$ as it is the most favorable given the background magnetic order. Other pairing symmetries can be treated similarly.

The superconducting order overlaps with the fluctuationinduced spiral modulation so we must construct superconducting order that can be treated self-consistently in the 
order-by-disorder scheme. The natural choice is a $p$-wave state in the basis that is diagonal in the presence of the background spiral, i.e., in the basis where electron spins are polarized locally parallel or antiparallel with the background spiral order. This leads to a state in which the $d$ vector of the $p$-wave superconductor rotates in concert with the magnetization. The state may be referred to as a pair density wave as it involves modulation of both magnetic and superconducting order, or alternatively Larkin-Ovchinnikov-Fulde-Ferrell-like since it involves a modulation of the superconducting order parameter, but in its $\mathrm{SU}(2)$ rather than $U(1)$ sector.

As it stands, the quantum order by disorder procedure requires that the emergent order changes the mean-field dispersion. In the case of a contact repulsion, superconductivity does not have this effect. One way around this (used to study the possibility of nematic order in Ref. 20) is to include a field conjugate to the superconducting order. After calculating the appropriate free energy and its fluctuation corrections, a Legendre transformation recovers the Gibbs free energy. Here, we use an equivalent self-consistent approach. The general scheme is as follows: (i) After having first diagonalized the electron states in the magnetic background, we add and subtract a term

$$
\sum_{\mathbf{k}}\left(\Delta_{\mathbf{k}} \tilde{c}_{-\mathbf{k} \uparrow}^{\dagger} \tilde{c}_{\mathbf{k} \uparrow}^{\dagger}+\text { c.c. }\right)
$$

in the free energy, where $\tilde{c}_{\mathbf{k} \uparrow}^{\dagger}$ creates a state with spin polarized parallel to the background magnetic order, which may be spiral in certain regions of the phase diagram. (ii) The terms $\mathcal{H}_{0}-\sum_{\mathbf{k}}\left(\Delta_{\mathbf{k}} \tilde{c}^{\dagger} \tilde{c}^{\dagger}+\right.$ c.c.) can be diagonalized by a Bogoliubov transformation. (iii) The contributions of the terms $\mathcal{H}_{\text {int }}+\sum_{\mathbf{k}}\left(\Delta_{\mathbf{k}} \tilde{c}^{\dagger} \tilde{c}^{\dagger}+\right.$ c.c. $)$ can be calculated perturbatively in the Bogoliubov basis. For BCS theory, the resulting free energy has the same gap equation as the conventional treatment. As we now show, using this approach in the case of a contact repulsion recovers spin-fluctuation mediated pairing. ${ }^{37-41}$

The superconducting order does not change the expectation of the contact interaction and so only enters the mean-field free energy through the kinetic term. Fluctuation corrections are obtained following the prescription developed for the fluctuation driven spin spiral; the fluctuation contributions to the free energy are calculated from Eq. (3), though now in the diagonal Bogoliubov basis. Overall, after expanding to order $|\Delta|^{2}$, we obtain the free energy ${ }^{47}$

$$
\begin{aligned}
\mathcal{F}(M, \Delta)= & -T \sum_{\mathbf{k}, \sigma} \ln \left(e^{-\xi_{\mathbf{k} \sigma} / T}+1\right)+\frac{g M^{2}}{2}-2 g^{2} \sum_{\substack{\mathbf{k}_{1}+\mathbf{k}_{2} \\
=\mathbf{k}_{3}+\mathbf{k}_{4}}} \frac{n_{\mathbf{k}_{1}}^{\uparrow} n_{\mathbf{k}_{2}}^{\downarrow}\left[n_{\mathbf{k}_{3}}^{\uparrow}+n_{\mathbf{k}_{4}}^{\downarrow}+\epsilon_{\mathbf{k}_{2}}^{\downarrow}-\epsilon_{\mathbf{k}_{3}}^{\uparrow}-\epsilon_{\mathbf{k}_{4}}^{\downarrow}\right.}{\epsilon_{\mathbf{k}}} \\
& -\sum_{\mathbf{k}} \frac{\left|\Delta_{\mathbf{k}}\right|^{2}}{2 \xi_{\mathbf{k}, \uparrow}}\left(2 n_{\mathbf{k}}^{\uparrow}-1\right)+g^{2} \sum_{\mathbf{k}, \mathbf{q}} \frac{\bar{\Delta}_{\mathbf{k}+\mathbf{q}}}{2 \xi_{\mathbf{k}+\mathbf{q}, \uparrow}}\left(1-2 n_{\mathbf{k}+\mathbf{q}}^{\uparrow}\right) \frac{\Delta_{\mathbf{k}}}{2 \xi_{\mathbf{k}, \uparrow}}\left(1-2 n_{\mathbf{k}}^{\uparrow}\right) \operatorname{Re} \chi^{\downarrow \downarrow}\left(\mathbf{q}, \epsilon_{\mathbf{k}+\mathbf{q}}^{\uparrow}-\epsilon_{\mathbf{k}}^{\uparrow}\right) \\
& +\sum_{\mathbf{k}} \frac{\left|\Delta_{\mathbf{k}}\right|^{2}}{2 \xi_{\mathbf{k}, \uparrow}}\left(1-2 n_{\mathbf{k}}^{\uparrow}\right) \partial_{\epsilon_{\mathbf{k}}} \operatorname{Re} \Sigma^{\uparrow}\left(\mathbf{k}, \epsilon_{\mathbf{k}}\right),
\end{aligned}
$$

where $\xi_{\mathbf{k} \sigma}=\epsilon_{\mathbf{k}}^{\sigma}-\mu \cdot \chi$ and $\Sigma$ are the susceptibility and self-energy evaluated at finite $M_{\mathbf{q}}$ and spiral vector $\mathbf{q}$, and are given by

$$
\operatorname{Re} \chi^{\downarrow \downarrow}(\mathbf{q}, \omega)=\sum_{\mathbf{p}} \frac{n_{\mathbf{p}}^{\downarrow}-n_{\mathbf{p}+\mathbf{q}}^{\downarrow}}{\epsilon_{\mathbf{p}+\mathbf{q}}^{\downarrow}-\epsilon_{\mathbf{p}}^{\downarrow}-\omega}
$$

and

$$
\partial_{\epsilon_{\mathbf{k}}} \Sigma^{\uparrow}\left(\mathbf{k}, \epsilon_{\mathbf{k}}\right)=-g^{2} \partial_{\epsilon_{\mathbf{k}}} \sum_{\mathbf{p}, \mathbf{q}} \frac{n_{\mathbf{p}-\mathbf{q}}^{\downarrow}\left(1-n_{\mathbf{k}-\mathbf{q}}^{\uparrow}\right)\left(1-n_{\mathbf{p}}^{\downarrow}\right)+\left(1-n_{\mathbf{p}-\mathbf{q}}^{\downarrow}\right) n_{\mathbf{k}-\mathbf{q}}^{\uparrow} n_{\mathbf{p}}^{\downarrow}-n_{\mathbf{p}-\mathbf{q}}^{\downarrow}}{\epsilon_{\mathbf{k}}^{\uparrow}+\epsilon_{\mathbf{p}-\mathbf{q}}^{\downarrow}-\epsilon_{\mathbf{k}-\mathbf{q}}^{\uparrow}-\epsilon_{\mathbf{p}}^{\downarrow}} .
$$

The first line of Eq. (8) that is independent of $\Delta$ is precisely the free energy of the ferromagnet derived earlier. At finite $\Delta$, the first term of the second line contains the mean-field response of the Fermi surface to Cooper pairing, and the subsequent terms arise from the fluctuation corrections.

The gap equation resulting from this is given by exactly the same expression as the Eliashberg equations obtained in spin-fluctuation theory. The resulting transition temperature for the pair density wave can be written as ${ }^{37-41}$

$$
T_{\mathrm{c}}=\frac{2 \mu^{\uparrow} e^{\mathcal{C}}}{\pi} \exp \left[-\frac{\left[1-\partial_{\epsilon} \operatorname{Re} \Sigma^{\uparrow}\left(\mathbf{k}, \epsilon_{\mathbf{k}}\right)\right]\left|\left\langle\theta_{\mathbf{k}}^{2}\right\rangle\right\rangle}{\left\langle\left\langle\theta_{\mathbf{k}+\mathbf{q}} \theta_{\mathbf{k}} \operatorname{Re} \chi^{\downarrow \downarrow}\left(\mathbf{q}, \epsilon_{\mathbf{k}+\mathbf{q}}-\epsilon_{\mathbf{k}}\right)\right\rangle\right\rangle}\right],
$$

where $\mathcal{C}$ is the Euler constant, and $\theta_{\mathbf{k}}$ is the angular dependence of the superconducting order parameter. ${ }^{48}$ Equation (10) gives the transition temperature in paramagnetic, ferromagnetic, and spiral phases provided $\chi$ and $\Sigma$ are calculated in the appropriate backgrounds. We note that both the fluctuationinduced pairing and field renormalization occur automatically in this approach. The field renormalization/self-energy term written here includes the effect of the regularization described above Eq. (3). Because of the intervention of phase reconstruction before the quantum critical point is reached, we do not require that the magnetic fluctuations take their critical form. ${ }^{37-41}$ 
The dependence of $\Sigma$ and $\chi$ upon the background magnetic order through the mean-field dispersion, $\epsilon_{\mathbf{k}}^{\sigma}(M, \mathbf{q})=$ $\epsilon_{\mathbf{k}}+\sigma \sqrt{(g M)^{2}+\left(\theta_{\mathbf{k}} \cdot \mathbf{q}\right)^{2}}$, induces cross terms between the superconducting and magnetic order parameters and their gradients. Indeed, such cross terms also appear in the meanfield expansion of the bare Cooper susceptibility and ultimately in the prefactor of chemical potential in Eq. (10). Terms arising from the fluctuation corrections are precisely the mode-mode coupling terms included by Kirkpatrick et al. previously. ${ }^{40}$

In practice, we find that although the gradient terms are crucial in driving the pair density wave state, expanding the susceptibility $\chi$ and self-energy $\Sigma$ in the spiral wave vector leads to subleading corrections that do not change the superconducting transition temperature appreciably. At small and large magnetization, we find

$$
\begin{aligned}
g^{2} & \left.\left\langle\left\langle\theta_{\mathbf{k}+\mathbf{q}} \theta_{\mathbf{k}} \operatorname{Re} \chi^{\downarrow \downarrow}\left(\mathbf{q}, \epsilon_{\mathbf{k}+\mathbf{q}}-\epsilon_{\mathbf{k}}\right)\right\rangle\right\rangle /\left\langle\theta_{\mathbf{k}}^{2}\right\rangle\right\rangle \\
& \approx \begin{cases}\frac{\left(g \nu_{\mathrm{F}}\right)^{2}}{5}\left[2 \ln 2-1+\frac{5}{3} \frac{g M}{\mu}(7-4 \ln 2)\right] & \text { small } M, \\
\frac{\left(g \nu_{\mathrm{F}}\right)^{2}}{2}\left(1-\frac{g M}{\mu}\right)^{3 / 2}\left(1+\frac{1}{3} \ln \left[2-2 \frac{g M}{\mu}\right]\right) & \text { large } M,\end{cases}
\end{aligned}
$$

and

$$
\left\langle\langle \partial _ { \epsilon _ { \mathbf { k } } } \Sigma ( \mathbf { k } , \epsilon _ { \mathbf { k } } ) \rangle \approx \left\{\begin{array}{ll}
\left(g \nu_{\mathrm{F}}\right)^{2}\left[\left(1-\frac{g M}{\mu}\right) \ln 2+\frac{1}{2}\right] & \text { small } M, \\
\frac{\left(g \nu_{\mathrm{F}}\right)^{2}}{2^{3 / 2}} \sqrt{1-\frac{g M}{\mu}} & \text { large } M .
\end{array}\right.\right.
$$

\section{PHASE DIAGRAM}

The phase diagram is shown in Fig. 1. With a first-order transition and spin spiral emerging at low temperatures, the phase diagram is a refinement of that presented in Ref. 35, the one notable difference being the order of the transition from the paramagnet to the spin spiral. In Ref. 35 only the lowest-order term in the free-energy expansion was calculated, implicitly assuming a second-order paramagnet-spiral transition. Here we have extended the free-energy expansion to order $M^{6}$, which shows that the paramagnet-spiral transition is in fact first order. ${ }^{20}$ Though this phase diagram is consistent with variational Monte Carlo results, ${ }^{35}$ the spiral phase here occupies a smaller range of interaction strengths due to the absence of subleading divergences. However, the topology of the phase diagram is identical so we proceed with the amenable Ginzburg-Landau formalism. As the superconductivity is weak it may be laid over the magnetic phase diagram without appreciable feedback.

Magnetic fluctuations drive Cooper pairing, so that even at $M=0$ a superconducting phase is formed. When the superconductivity overlaps regions of the phase diagram supporting magnetic order, there is a large increase in the transition temperature because of the mode-mode coupling effects described above and in Ref. 40. The transition temperature tails off exponentially to zero when the magnetization has depleted the number of pairing (spin-down) electrons to zero, thus sending the susceptibility term to zero. In Fig. 1, the superconducting transition temperature shows a first-order jump upon passing into the magnetically ordered phase, tracking the local magnetization. Where the superconductivity overlaps the spiral phase, its $d$ vector is locked to the local magnetic order, forming the pair density wave.

\section{DISCUSSION}

The minimal Hamiltonian Eq. (1) —an electron gas with contact repulsion-delivers a remarkably rich phase diagram when all possible fluctuations are accounted for. Fluctuations drive the itinerant ferromagnet near to several possible instabilities. Transverse fluctuations can drive a first-order ferromagnetic transition or a spin spiral phase. Longitudinal magnetic fluctuations drive a $p$-wave superconducting instability. These instabilities overlap forming a pair density wave. Comparison with variational Monte Carlo calculations ${ }^{35}$ shows that the quantum order-by-disorder approach, as well as revealing the underlying physics in a transparent manner, can accurately determine the phase boundaries.

However, the model may require modification in more realistic settings. First, unlike the local interaction used here, the screened Coulomb interaction has finite range and is sampled at different momenta by mean-field and fluctuation contributions to the free energy. The tricritical point-the lowest temperature at which a continuous ferromagnetic to paramagnet transition is observed-is commonly seen at temperatures $\sim 0.02 T_{\mathrm{F}}$ (Ref. 17) that are significantly lower than the prediction of the minimal Hamiltonian $\sim 0.3 T_{\mathrm{F}}$. However, it has been shown both with variational Monte Carlo and analytically that a finite ranged screened Coulomb interaction reduces the tricritical temperature found in a quantum order-by-disorder calculation to the realistic experimental range of $\sim 0.02 T_{\mathrm{F}}{ }^{49}$ Second, real band structures, with deviations from quadratic electron dispersions, shift the subtle balance between the near multicritical phases. ${ }^{20}$ Finally, spin-orbit induced magnetic anisotropy can suppress transverse magnetic fluctuations relative to the longitudinal fluctuations so changing the balance between magnetic and superconducting instabilities, ${ }^{38}$ and may ultimately invalidate the neglect of backreaction of superconducting order upon the magnetic order.

The necessity of these modifications to the model Hamiltonian makes direct comparison of our results with experiment delicate. The types of order predicted in our model calculations have all been seen in (or suggested by) experiment. For example, $p$-wave superconducting is found in $\mathrm{UGe}_{2},{ }^{21-23}$ URhGe ${ }^{24}$ and UCoGe. ${ }^{25}$ In the magnetic state $\mathrm{UGe}_{2}$ displays superconductivity below $T_{\mathrm{c}} \approx 500 \mathrm{mK}$, and no superconductivity has been observed on the paramagnetic side. Theory predicts that the peak superconducting temperature has a ratio of 200:1 between the ferromagnetic and paramagnetic sides, meaning that on the paramagnetic side $\mathrm{UGe}_{2}$ would have a transition temperature at $T_{\mathrm{c}} \approx 3 \mathrm{mK}$ that is too small to measure, thus maintaining consistency with experiment. ${ }^{40}$ Evidence for spin spiral behavior, though less direct, is found in $\mathrm{CeFePO},{ }^{14} \mathrm{NbFe}_{2},{ }^{15} \mathrm{Yb}\left(\mathrm{Rh}_{0.73} \mathrm{Co}_{0.27}\right)_{2} \mathrm{Si}_{2},{ }^{16}$ and possibly $\mathrm{Sr}_{3} \mathrm{Ru}_{2} \mathrm{O}_{7} \cdot{ }^{17,18}$ Although evidence for the coexistence of superconductivity and spiral magnetism has not yet been found, it could be measured through a drop of electrical resistance coupled with the emergence of spatial texture in a neutron scattering study. Nevertheless, we take the occurrence of these ingredients as reason for positive expectation. 
The quantum order-by-disorder framework provides a unifying and physical reformulation of diagrammatic approaches to particle-hole and particle-particle instabilities. The Fermi surface provides boundary conditions for fluctuations through Pauli exclusion. Modification of the Fermi surface by the introduction of order affects the spectrum of fluctuations, and can be self-consistently stabilized by them. We have shown that this drives the formation of a different phase, the pair density wave. This formalism guides future calculations in a direction that may be fruitful in a variety of situations.

\section{ACKNOWLEDGMENTS}

We are grateful to Una Karahasanovic and Frank Krüger for useful discussions, and acknowledge Gonville \& Caius College and the EPSRC (Grant No. EP/I004831/1) for financial support. *gjc29@cam.ac.uk

†andrew.green@ucl.ac.uk

${ }^{1}$ S. L. Sondhi, S. M. Girvin, J. P. Carini, and D. Shahar, Rev. Mod. Phys. 69, 315 (1997).

${ }^{2}$ P. Coleman and A. J. Schofield, Nature (London) 433, 226 (2005).

${ }^{3}$ S. Sachdev, Quantum Phase Transitions, 2nd ed. (Cambridge University Press, Cambridge, UK, 1999).

${ }^{4}$ J. Hertz, Phys. Rev. B 14, 1165 (1976).

${ }^{5}$ D. Belitz, T. R. Kirkpatrick, and T. Vojta, Phys. Rev. B 55, 9452 (1997).

${ }^{6}$ D. Belitz, T. R. Kirkpatrick, and T. Vojta, Phys. Rev. Lett. 82, 4707 (1999).

${ }^{7}$ D. V. Efremov, J. J. Betouras, and A. Chubukov, Phys. Rev. B 77, 220401 (2008).

${ }^{8}$ D. L. Maslov and A. V. Chubukov, Phys. Rev. B 79, 075112 (2009).

${ }^{9}$ J. Rech, C. Pepin, and A. V. Chubukov, Phys. Rev. B 74, 195126 (2006).

${ }^{10}$ T. R. Kirkpatrick and D. Belitz, Phys. Rev. B 85, 134451 (2012).

${ }^{11}$ A. J. Millis, Phys. Rev. B 48, 7183 (1993).

${ }^{12}$ T. Moriya, Solid State Science, Vol. 56 (Springer, Berlin, 1985).

${ }^{13}$ D. Belitz and T. R. Kirkpatrick, arXiv:1204.0873.

${ }^{14}$ S. Lausberg et al., Phys. Rev. Lett. 109, 216402 (2012).

${ }^{15}$ D. A. Tompsett, R. J. Needs, F. M. Grosche, and G. G. Lonzarich, Phys. Rev. B 82, 155137 (2010).

${ }^{16}$ S. Lausberg et al., arXiv:1210.5463.

${ }^{17}$ R. Borzi, S. A. Grigera, J. Farrell, R. S. Perry, S. J. S. Lister, S. L. Lee, D. A. Tennant, Y. Maeno, and A. P. Mackenzie, Science 315, 214 (2007).

${ }^{18}$ A. M. Berridge, A. G. Green, S. A. Grigera, and B. D. Simons, Phys. Rev. Lett. 102, 136404 (2009).

${ }^{19}$ A. V. Chubukov and D. L. Maslov, Phys. Rev. Lett. 103, 216401 (2009).

${ }^{20}$ U. Karahasanovic, F. Krüger, and A. G. Green, Phys. Rev. B 85, 165111 (2012).

${ }^{21}$ S. Watanabe and K. Miyake, J. Phys. Chem. Solids 63, 1465 (2002).

${ }^{22}$ S. S. Saxena et al., Nature (London) 406, 587 (2000).

${ }^{23}$ A. Huxley, I. Sheikin, E. Ressouche, N. Kernavanois, D. Braithwaite, R. Calemczuk, and J. Flouquet, Phys. Rev. B 63, 144519 (2001).

${ }^{24}$ D. Aoki et al., Nature (London) 413, 613 (2001).

${ }^{25}$ N. T. Huy et al., Phys. Rev. Lett. 99, 067006 (2007).

${ }^{26}$ A. Gasparini et al., J. Low Temp. Phys. 161, 134 (2010).
${ }^{27}$ R. Balian and N. R. Werthamer, Phys. Rev. 131, 1553 (1963).

${ }^{28}$ P. Anderson and W. F. Brinkman, Phys. Rev. Lett. 30, 1108 (1973).

${ }^{29}$ W. F. Brinkman, J. W. Serene, and P. W. Anderson, Phys. Rev. A 10, 2386 (1974).

${ }^{30}$ A. I. Larkin and Yu. N. Ovchinnikov, Zh. Eksp. Teor. Fiz. 47, 1136 (1964) [Sov. Phys. JETP 20, 762 (1965)].

${ }^{31}$ P. Fulde and R. A. Ferrell, Phys. Rev. 135, A550 (1964).

${ }^{32}$ T. Neupert and M. Sigrist, J. Phys. Soc. Jpn. 80, 114712 (2011).

${ }^{33}$ G. J. Conduit and B. D. Simons, Phys. Rev. A 79, 053606 (2009).

${ }^{34}$ R. A. Duine and A. H. MacDonald, Phys. Rev. Lett. 95, 230403 (2005).

${ }^{35}$ G. J. Conduit, A. G. Green, and B. D. Simons, Phys. Rev. Lett. 103, 207201 (2009)

${ }^{36}$ F. Krüger, U. Karahasanovic, and A. G. Green, Phys. Rev. Lett. 108, 067003 (2012).

${ }^{37}$ D. Fay and J. Appel, Phys. Rev. B 22, 3173 (1980).

${ }^{38}$ N. D. Mathur et al., Nature (London) 394, 39 (1998).

${ }^{39}$ R. Roussev and A. J. Millis, Phys. Rev. B 63, 140504(R) (2001).

${ }^{40}$ T. R. Kirkpatrick, D. Belitz, T. Vojta, and R. Narayanan, Phys. Rev. Lett. 87, 127003 (2001).

${ }^{41}$ Z. Wang, W. Mao, and K. Bedell, Phys. Rev. Lett. 87, 257001 (2001).

${ }^{42}$ S. Coleman and E. Weinberg, Phys. Rev. D 7, 1888 (1973).

${ }^{43}$ J. Villain, R. Bidaux, J.-P. Carton, and R. Conte, J. Phys. (France) 41, 1263 (1980).

${ }^{44}$ H. B. G. Casimir and D. Polder, Phys. Rev. 73, 360 (1948).

${ }^{45}$ In writing this form of the fluctuation correction, we have used a one-loop regularization of the interaction as in Refs. 33 and 35, $g_{\mathbf{k}_{1}, \mathbf{k}_{2}} \rightarrow g-2 g^{2} \sum_{\mathbf{k}_{3}, \mathbf{k}_{4}}^{\prime}\left(\epsilon_{\mathbf{k}_{1}}^{\uparrow}+\epsilon_{\mathbf{k}_{2}}^{\downarrow}-\epsilon_{\mathbf{k}_{3}}^{\uparrow}-\epsilon_{\mathbf{k}_{4}}^{\downarrow}\right)^{-1}$.

${ }^{46}$ A. P. Mackenzie and Y. Maeno, Rev. Mod. Phys. 75, 657 (2003).

${ }^{47}$ In order to obtain the form of the free-energy term - the first term on the second line-we have integrated by parts with respect to $\epsilon_{\mathbf{k}}$ assuming that we may linearize at the Fermi surface. Notice that the self-energy has an additional, final term proportional to $n_{\mathbf{p}-\mathbf{q}}$ compared to usual. This arises because the regularization of the interaction parameter amounts to a field renormalization. This additional term—which arises automatically—avoids double counting this field renormalization.

${ }^{48}$ Angular brackets indicate angular averages over the Fermi surface, $\quad\left\langle\left\langle\theta_{\mathbf{k}}^{2} n_{\mathbf{k}}\right\rangle\right\rangle=\sum_{\mathbf{k}} \theta_{\mathbf{k}}^{2} n_{\mathbf{k}} \delta\left(\epsilon_{\mathbf{k}}-\mu\right) \quad$ and $\quad\left\langle\left\langle\theta_{\mathbf{k}+\mathbf{q}} \theta_{\mathbf{k}} n_{\mathbf{k}, \mathbf{q}}\right\rangle\right\rangle=$ $\sum_{\mathbf{k}, \mathbf{q}} \theta_{\mathbf{k}+\mathbf{q}} \theta_{\mathbf{k}} n_{\mathbf{k}, \mathbf{q}} \delta\left(\epsilon_{\mathbf{k}}-\mu\right) \delta\left(\epsilon_{\mathbf{k}+\mathbf{q}}-\mu\right)$.

${ }^{49}$ G. J. Conduit and C. W. von Keyserlingk, arXiv:1301.6036. 